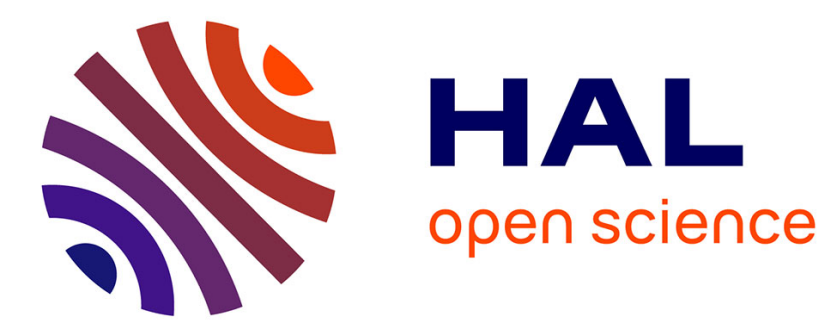

\title{
Les logiques plurielles d'une très faible participation : retour sur les scrutins auprès des salariés des très petites entreprises
}

Tristan Haute

\section{- To cite this version:}

Tristan Haute. Les logiques plurielles d'une très faible participation : retour sur les scrutins auprès des salariés des très petites entreprises. La Revue de l'IRES, 2020, La Revue de l'IRES, n $101-102$ (2), p. 3-27. 10.3917/rdli.101.0003 . hal-03336548

\section{HAL Id: hal-03336548 \\ https://hal.univ-lille.fr/hal-03336548}

Submitted on 7 Sep 2021

HAL is a multi-disciplinary open access archive for the deposit and dissemination of scientific research documents, whether they are published or not. The documents may come from teaching and research institutions in France or abroad, or from public or private research centers.
L'archive ouverte pluridisciplinaire HAL, est destinée au dépôt et à la diffusion de documents scientifiques de niveau recherche, publiés ou non, émanant des établissements d'enseignement et de recherche français ou étrangers, des laboratoires publics ou privés. 


\title{
Les logiques plurielles d'une très faible participation : retour sur les scrutins auprès des salariés des très petites entreprises
}

\author{
Tristan HAUTE ${ }^{1}$
}

En France, deux scrutins ont été organisés en 2012 et en 2017 auprès des salariés des très petites entreprises (TPE, moins de 11 salariés) pour compléter la mesure de la représentativité syndicale dans le secteur privé. Or, ces votes sur sigles, sans élu, par Internet ou par correspondance n'ont absolument pas mobilisé les salariés (10,4\% de participation en 2012 et 7,3\% en 2017).

Alors que plusieurs travaux se sont intéressés à la mise en place difficile de ces scrutins et aux pratiques syndicales déployées pour mobiliser les salariés des TPE, cet article propose différentes pistes explicatives complémentaires de la très faible participation électorale enregistrée. Celle-ci s'explique moins par les opinions des salariés que par des facteurs structurels, à savoir les spécificités de ce scrutin sans enjeu, les caractéristiques sociales et économiques des salariés des TPE et la faiblesse des implantations et des contacts syndicaux dans les TPE. Certaines branches d'activité où les enjeux du vote sont un peu plus saillants et où des syndicats corporatifs développent des activités de service, comme la branche des assistantes maternelles, sont d'ailleurs marquées par une mobilisation électorale significativement plus importante.

Le vote tient une place grandissante dans le système français de relations professionnelles. Ainsi, des scrutins auprès des salariés du particulier employeur et des entreprises ou associations de moins de 11 salariés, dits

1. Docteur en science politique, Université de Lille, CERAPS, UMR 8026 CNRS, tristan.haute@ univ-lille.fr. Cet article est issu de notre travail de thèse (Haute, 2019a). Nous tenons à remercier nos directeurs, Jean-Gabriel Contamin et Karel Yon, ainsi que Kevin Guillas-Cavan et le comité de rédaction de la revue pour leurs nombreux conseils et relectures. 
scrutins TPE, ont été instaurés par la loi du 15 octobre 2010 qui complète le dispositif de mesure de la représentativité syndicale créé par la loi du 20 août 2008 portant « rénovation de la démocratie sociale ». Avec cette dernière réforme, les résultats des élections professionnelles déterminent désormais la représentativité des organisations syndicales, leur capacité à participer aux négociations collectives et leur poids dans celles-ci ${ }^{2}$.

Les scrutins TPE sont organisés nationalement et les salariés, qui reçoivent un matériel de vote à domicile, sont appelés à voter par correspondance ou par Internet pour des sigles syndicaux. Jusqu'en 2017, aucun élu n’était directement désigné par les salariés. C'est pourquoi nous parlons de scrutins TPE et non d'élections TPE ${ }^{3}$. Le seul objectif de ces scrutins est de sécuriser juridiquement le dispositif de mesure d'audience pour le calcul de la représentativité syndicale. Ils permettent de ne pas exclure de cette mesure d'audience les salariés des TPE qui n'ont pas la possibilité de demander l'organisation d'élections de représentants du personnel dans leur entreprise. Dans la pratique, les voix obtenues par les organisations syndicales lors des scrutins TPE sont additionnées avec celles obtenues lors des élections de représentants du personnel en entreprise et lors des élections dans les chambres d'agriculture pour les salariés des exploitations agricoles. Cette addition se fait sans aucune pondération : il n'est pas tenu compte, lors du calcul, de la part des salariés des TPE ou des salariés des exploitations agricoles au sein du salariat et seuls les nombres de suffrages exprimés sont pris en compte. Cette addition permet d'obtenir, tous les quatre ans, une mesure d'audience pour le calcul de la représentativité syndicale au niveau interprofessionnel mais aussi au sein de chaque branche professionnelle (Haute, Yon, 2018). Un premier scrutin TPE a donc eu lieu en novembre et en décembre 2012 pour compléter la mesure d'audience du cycle 2009-2012 et un second a eu lieu en janvier 2017 pour parachever la mesure d'audience du cycle 2013-2016.

Les deux scrutins TPE ont été marqués par une très faible participation des salariés. Ainsi, seuls 10,4\% des 4,6 millions de salariés inscrits ont voté en 2012 et seuls 7,3\% des 4,5 millions d'inscrits ont voté en 2017. Cette faible participation n'a pas surpris les syndicalistes et les chercheurs, tant ces scrutins sont, comme nous l'avons souligné précédemment, artificiels et sans autre enjeu que de compléter la mesure de la représentativité syndicale (Andolfatto, 2017). Rappelons de plus que le scrutin de janvier 2017, initialement prévu entre le 28 novembre et le 12 décembre 2016, a été décalé suite à un contentieux juridique (voir infra, tableau 3). L'ensemble du matériel de vote et de propagande a dû être envoyé de nouveau en urgence.

2. Sur cette réforme, nous renvoyons au troisième chapitre de l'ouvrage Sociologie politique du syndicalisme (Giraud et al., 2018:77-107).

3. La loi Rebsamen du 17 août 2015 a toutefois créé des commissions paritaires régionales interprofessionnelles (CPRI), mais celles-ci n'étaient pas encore en place lors du scrutin de 2017. 
Le décalage explique sans doute, au moins en partie, le recul de la participation entre 2012 et 2017 et rend les deux scrutins difficilement comparables.

Le faible nombre de votants aux scrutins TPE rend ceux-ci encore moins significatifs en matière de représentativité syndicale. En effet, si les salariés des TPE représentent près d'un quart du salariat du secteur privé, leurs votes représentent moins de $10 \%$ des suffrages exprimés pris en compte pour calculer la représentativité syndicale dans le secteur privé (Haute, Yon, 2018:111). Cette marginalité dans le dispositif de quantification de la représentativité syndicale explique sans doute en partie la faible investigation des résultats de ces scrutins.

Un certain nombre de travaux se sont déjà intéressés aux scrutins TPE et notamment à la mise en place de ce dispositif original (Rey, 2014, 2016 ; Andolfatto, 2017) et aux pratiques syndicales de mobilisation électorale des salariés lors de ces scrutins (Béroud, Guillaume, 2013; Rey et al., 2014). L'analyse des résultats des scrutins TPE reste cependant un point aveugle auquel cet article entend contribuer en proposant une première analyse des facteurs de la faible participation enregistrée.

S'intéresser à la participation des salariés aux scrutins TPE a un triple intérêt. En premier lieu, ces scrutins concernent tout de même un quart du salariat du secteur privé en France et ils constituent pour les salariés concernés l'unique moment potentiel de rencontre avec le vote professionnel et, plus généralement, l'une des rares opportunités de rencontre avec le syndicalisme. En second lieu, si le poids des voix issues du scrutin TPE est marginal au niveau interprofessionnel, cela n'est pas le cas dans des branches où les salariés des TPE sont largement majoritaires. Dans ces branches, les résultats des scrutins TPE déterminent la représentativité, la capacité à négocier et le poids dans la négociation collective des différentes organisations syndicales (Haute, Yon, 2018:111). En troisième lieu, une analyse des facteurs de l'abstention permet de mettre à l'épreuve l'hypothèse d'une défiance des salariés à l'égard des syndicats dont l'abstention serait un indicateur.

Cependant, si nous mobilisons une pluralité de matériaux ainsi qu'une littérature importante relative aux relations professionnelles dans les petites entreprises, notre analyse se veut avant tout exploratoire du fait de la fragilité de nos matériaux (encadré 1). Nous proposons ainsi moins des conclusions définitives que des pistes de réflexion.

Comme l'ont souligné un certain nombre de commentaires, à commencer par ceux des organisations syndicales adressés à la DGT (encadré 1), la faible participation aux scrutins TPE est tout d'abord à mettre en lien avec deux spécificités de ce scrutin : des conditions matérielles du vote peu favorables à la mobilisation et l'absence d'enjeu aux yeux de la très grande majorité des salariés (I). Cependant, d'autres logiques structurelles 


\section{Présentation des matériaux utilisés}

L'étude de la participation aux scrutins TPE est fortement contrainte par le manque de données disponibles. En effet, aucune enquête robuste, réalisée au niveau individuel, ne s'est intéressée au vote des salariés aux scrutins TPE. L'enquête Relations professionnelles et négociations d'entreprise (REPONSE) ne concerne que les salariés des établissements de 11 salariés ou plus. De même, les vagues de l'enquête Statistiques sur les ressources et conditions de vie (SRCV) qui interrogent les salariés sur leur vote aux élections professionnelles ne mentionnent pas la taille de l'entreprise, ce qui ne permet pas d'isoler les salariés des TPE parmi les répondants. Notre démarche a dès lors été de croiser des matériaux qualitatifs et quantitatifs qui, pris isolément, s'avèrent lacunaires.

Quatre types de matériaux sont utilisés dans cet article. Nous nous sommes tout d'abord appuyé sur les données électorales agrégées au niveau des branches professionnelles. Les champs conventionnels, s'ils sont le produit de constructions sociopolitiques, processus dans lesquels l'intervention du patronat est centrale (Haute, Yon, 2018:110), fournissent une diversité de configurations socioéconomiques. Les données disponibles nous permettent d'exploiter cette diversité en testant l'existence de relations entre les caractéristiques du salariat et la participation au sein de chaque branche. Nous avons plus précisément mis en relation la participation au scrutin TPE de 2012 avec des données économiques et sociales issues des fiches statistiques 2012 de la Direction de l'animation, de la recherche, des études et des statistiques (Dares) du ministère du Travail. L'appariement a pu être réalisé pour 231 branches professionnelles, soit $54,9 \%$ du corps électoral du scrutin TPE de $2012{ }^{1}$. Toutefois, les indicateurs relatifs aux très petites entreprises concernent les entreprises de un à neuf salariés dans les données de la Dares alors que, dans le cadre du scrutin TPE, les TPE sont les entreprises de un à dix salariés. De plus, l'analyse de données au niveau agrégé pose un certain nombre de problèmes méthodologiques. En effet, l'observation de relations statistiques au niveau collectif ne signifie pas que celles-ci sont vérifiées au niveau individuel.

Pour compléter cette analyse au niveau agrégé, nous avons réalisé une modeste enquête par questionnaire auprès de salariés des TPE. Le questionnaire a été diffusé par courriel et sur les réseaux sociaux auprès d'organisations syndicales ou patronales, sur des groupes professionnels et dans notre entourage avec comme objectif de maximiser le nombre de répondants. Ce mode de diffusion ne permet toutefois pas d'estimer avec précision le nombre de personnes qui ont effectivement reçu le questionnaire. De plus, ce questionnaire était dès lors sans but représentatif. Les résultats ont avant tout permis d'illustrer et de préciser des relations statistiques observées au niveau agrégé. Le questionnaire interrogeait les salariés sur leur connaissance du scrutin, sur leur vote et sur leur rapport au syndicalisme. Parallèlement, était posée une série de questions sur leurs caractéristiques sociales et 
professionnelles (genre, âge, ancienneté dans l'entreprise, conditions d'emploi et de travail...). 72 salariés ont répondu entre janvier et septembre 2018, un échec qui illustre le difficile contact avec le salariat des TPE.

Nous avons également mobilisé cinq entretiens réalisés avec des responsables de structures territoriales de la CGT, de I'UNSA et de Solidaires ayant participé aux campagnes TPE. Les entretiens ont été menés entre le 30 novembre et le 18 décembre 2017. Les enquêtés étaient interrogés sur les dispositifs mis concrètement en place dans leur département lors de la campagne TPE et sur les difficultés rencontrées pour mobiliser les salariés.

Nous avons enfin utilisé les contributions des organisations syndicales remises à la Direction générale du travail (DGT) à l'issue de chacun des deux cycles électoraux pour établir un bilan de la mesure de la représentativité syndicale. Ces contributions dressent en particulier des constats relativement convergents sur l'organisation pratique des scrutins TPE.

1. À noter que les branches des salariés du « particulier employeur » et des assistantes maternelles du « particulier employeur » (voir infra, encadré 4), qui représentent à elles deux près de $20 \%$ des inscrits, ne sont pas incluses dans ce fichier, faute de données socioéconomiques disponibles.

sont également à l'œuvre. Ainsi, la faible participation des salariés des TPE s'explique en partie par leurs caractéristiques sociales et professionnelles, une faible rémunération et une faible intégration professionnelle favorisant l'abstention (II). De même, la faible mobilisation électorale est également le résultat d'une distance, plus pratique qu'idéologique, entre les salariés des TPE et les organisations syndicales : ce sont d'ailleurs les salariés directement en contact avec le syndicalisme, notamment au sein de groupes professionnels fortement structurés par des syndicats corporatifs et de service, qui sont les plus participants électoralement (III).

\section{L'impact des spécificités du scrutin TPE sur la participation}

L'abstention massive enregistrée en 2012 et en 2017 n'a pas surpris les syndicalistes et les chercheurs du fait des spécificités des scrutins TPE (Andolfatto, 2017). Le recours au vote électronique, socialement sélectif, et surtout l'ampleur des phénomènes de non-inscription et de mal-inscription n’ont ainsi pas été propices à la participation (I.1). Surtout, les enjeux potentiellement saillants de ces scrutins sont peu nombreux puisque le vote ne débouche pas sur l'élection de représentants, n'influence pas les relations sociales quotidiennes en entreprise et, hormis dans certaines branches, ne modifie pas les équilibres syndicaux issus des élections en entreprise (I.2).

\section{I.1. Des conditions matérielles du vote peu propices à la participation}

Toutes les organisations syndicales ont déploré, outre le décalage du scrutin de 2017 de plusieurs semaines du fait d'un contentieux juridique 
(voir supra) et une couverture médiatique inexistante ${ }^{4}$, des conditions de déroulement du vote peu favorables à la mobilisation des salariés des TPE.

Premièrement, alors que le vote se déroule en partie sur Internet, ce mode de participation implique une forme de "sélectivité sociale » (Béroud, Dompnier, 2013:526). Ce sont les salariés qui ont l'habitude d'utiliser des outils numériques variés qui sont les plus à même de s'approprier une procédure de vote en ligne que nombre de syndicalistes rencontrés jugent complexe. C'est du moins ce qui ressort de nos différents entretiens avec des responsables syndicaux. Si la possibilité de voter par correspondance existe (celle-ci étant utilisée par deux tiers des votants), sa disparition potentielle en 2021 avec le passage à un vote exclusivement par Internet fait craindre aux syndicats une nouvelle baisse de la participation, à l'image de ce qui a été observé dans l'Éducation nationale (Haute, 2019b:158).

Deuxièmement, la construction de la liste électorale nationale implique que tous les salariés des TPE au moment du scrutin ne sont pas appelés à voter. En effet, la liste électorale ne comprend que les salariés au 31 décembre de l'année précédant le scrutin alors que celui-ci se déroule à la fin de l'année. Même si des rectifications sont possibles, ce mode de construction de la liste électorale « exclut nombre de salariés qui ont été salariés d'une TPE dans l'année, mais pas ou plus au 31 décembre » ${ }^{5}$, ce qui affecte particulièrement la représentativité du corps électoral dans les secteurs où les salariés saisonniers sont nombreux ou dans les métiers du spectacle. La rectification de la liste est certes possible, mais nécessite une démarche individuelle et peut se faire durant un intervalle de temps de quelques semaines seulement, ce que regrette l'ensemble des organisations syndicales. Notons toutefois que cette problématique de la construction de la liste électorale était déjà présente lors des élections prud'homales auxquelles les salariés des TPE pouvaient voter jusqu'en 2008 (Dompnier, 2018:463).

Troisièmement, même lorsqu'ils sont inscrits, des salariés se retrouvent dans l'impossibilité de voter car ils n'ont pas reçu à leur domicile le matériel permettant de voter par correspondance ou en ligne. Les changements d'habitation entre la constitution de la liste électorale (au 31 décembre de l'année précédente) et le scrutin ne peuvent d'ailleurs être pris en compte. Les organisations syndicales signalent plus généralement de nombreux soucis dans l'acheminement du matériel de vote. L'union syndicale Solidaires déclare ainsi avoir envoyé sa propagande en utilisant la liste électorale fournie par le ministère «à quelques secteurs professionnels ou départements

4. Pour une synthèse plus exhaustive, voir D. Perais, «Élections professionnelles dans les très petites entreprises (TPE) : circulez, y a rien à voir ! », Acrimed - Action critique médias, 9 mars 2017, http://www.acrimed.org/Elections-professionnelles-dans-les-tres-petites.

5. Contribution de la CGT à la DGT, 26 février 2018. 
ciblés » et elle constate que « le taux de retour pour adresse incorrecte a avoisiné les $20 \%{ }^{6}$.

Au-delà des soucis de réception du matériel de vote, les organisations syndicales ont relevé que de nombreux salariés se sont retrouvés inscrits dans des collèges ou dans des branches erronés. Ainsi, la CFE-CGC indique que, pour le scrutin de 2017, il a été procédé au retrait de 90000 salariés répertoriés comme cadres par erreur, ce qui représente près de $23 \%$ du corps électoral du collège " cadres " ${ }^{7}$. De la même manière, le Syndicat national des journalistes (SNJ) a observé que, en 2012, parmi les électeurs rattachés à la convention collective des journalistes, seuls $60 \%$ étaient effectivement journalistes ${ }^{8}$. Cette mal-inscription, si elle ne constitue pas, en soi, un obstacle à la participation, peut modifier l'offre électorale à laquelle les salariés sont confrontés ?

\section{I.2. La difficile émergence d'enjeux électoraux saillants}

Comme l'indiquent nos données d'enquête, une partie non négligeable des abstentionnistes justifient leur abstention par le fait qu'ils «ne voyaient pas l'utilité de ce vote». Cela concerne notamment des salariés par ailleurs plutôt prédisposés à participer (aux conditions d'emploi stables, ayant des contacts avec les syndicats ou ayant une bonne opinion des syndicats) (voir infra, III.2). On peut ici mentionner l'exemple d'un agent administratif de 29 ans, salarié dans le secteur associatif, diplômé de l'enseignement supérieur (bac +4 ou plus) et se positionnant très à gauche. Il déclare des contacts avec les syndicats, des discussions avec des militants ainsi que la participation à des actions collectives (hors arrêt de travail). Mais, s'il a eu connaissance du scrutin par des tracts et des affiches, il n'a pas voté, considérant que le vote était inutile et qu'il ne connaissait pas les candidats.

Alors que les scrutins TPE ne débouchent pas, pour l'heure, sur la désignation d'élus, l'émergence d'une représentation au niveau régional avec la mise en place des commissions paritaires régionales interprofessionnelles (CPRI) ne devrait pas modifier significativement la situation. D'abord, le périmétrage régional maintient de fait un éloignement entre les syndicats et les salariés des TPE. Ensuite, le nombre de représentants est plutôt faible (dix par région). Enfin, on peut douter de la capacité de décision de ces commissions dans un contexte où une partie des organisations patronales continuent de s'appuyer sur un « esprit de la boutique et du petit commerce [qui] serait basé sur la confiance réciproque et devrait se passer de toute ingérence syndicale » (Rey et al., 2014:170). Ainsi, si de

6. Contribution de Solidaires à la DGT, 20 décembre 2017.

7. Contribution de la CFE-CGC à la DGT, février 2018.

8. Contribution du SNJ à la DGT, 15 novembre 2013.

9. Par exemple, il faut être dans le collège "cadres » pour pouvoir voter pour la CFE-CGC. De même, certains syndicats professionnels ne sont candidats qu'au sein d'une convention collective. 
telles commissions paritaires ont vu le jour à partir de 2010 dans l'artisanat (Gassier, 2020), une analyse au niveau agrégé montre que la couverture des salariés des TPE par une commission paritaire régionale interprofessionnelle de l'artisanat (CPRIA) n'influence aucunement leur participation électorale (voir infra, encadré 2). De même, les acteurs syndicaux insistent moins sur leur action dans les CPRIA que sur le rôle des conseillers prud'homaux. Alors que le nombre de conseillers dont dispose chaque organisation dépend de ses résultats électoraux dans l'ensemble du secteur privé, des syndicalistes s'efforcent d'incarner le vote en affirmant que « plus de voix » pour une organisation, c'est plus de conseillers prud'homaux issus de ses rangs pour défendre les salariés ${ }^{10}$.

De plus, même si les voix issues des scrutins TPE participent au calcul de la représentativité syndicale, elles comptent peu au niveau interprofessionnel (voir supra). Si cette situation de marginalité est bien moins vérifiée quand on passe au niveau des branches professionnelles, rappelons qu'un nombre non négligeable de salariés ne sont pas couverts par une convention collective, notamment dans certains secteurs d'activité où les TPE sont majoritaires. Les salariés des TPE sont cependant majoritaires, voire hégémoniques, au sein d'un grand nombre de branches professionnelles dont certaines regroupent des effectifs importants. C'est par exemple le cas de la convention collective des salariés du « particulier employeur » (667426 salariés inscrits en 2017), de la convention collective des assistantes maternelles ${ }^{11} \mathrm{du}$ " particulier employeur» (286326 salariés inscrits en 2017) ou d'un certain nombre de branches du commerce et des services (boulangerie, pharmacie, coiffure, immobilier, cabinets médicaux...). Dans ces branches, le vote des salariés des TPE fixe la représentativité des différentes organisations syndicales et les équilibres syndicaux lors des négociations collectives. Si cet enjeu conventionnel se limite à certaines branches, il peut s'avérer saillant aux yeux des salariés concernés. En effet, la convention collective constitue souvent le seul texte juridique auquel les salariés font référence (Farvaque, Lefebvre, 2010:18 ; Trémeau, 2017:180). Néanmoins, il n'existe pas de relation statistique entre la part des salariés des TPE et la participation électorale au sein d'une branche (voir infra, tableau 1). En d'autres termes, l'influence des résultats du scrutin TPE sur la mesure de la représentativité syndicale au niveau conventionnel n'est pas, en soi, un facteur de la participation.

Si les conditions matérielles du vote et l'absence d'enjeu saillant aux yeux de la plupart des salariés ne favorisent pas leur mobilisation électorale, cette logique explicative de l'abstention apparait insuffisante. Si la

10. Entretien de l'auteur avec un responsable territorial CGT, 30 novembre 2017.

11. Pour cette profession, nous utilisons le féminin par convention puisque moins de $1 \%$ des demandes d'agrément sont faites par des hommes. 
participation aux scrutins TPE est bien inférieure à celle enregistrée lors des élections de représentants du personnel dans les entreprises de 11 à 49 salariés (47,6 \% selon l'enquête REPONSE 2017), rappelons en effet que les salariés des TPE votaient déjà très peu lors des élections prud'homales (Blanchard et al., 1996:60). Il convient dès lors d'explorer la pertinence d'autres logiques explicatives. Ainsi, au-delà des spécificités des scrutins TPE, nous proposons de nous intéresser aux spécificités des salariés des TPE par rapport au reste du salariat.

\section{Une faible participation liée aux caractéristiques sociales des salariés des TPE}

L'abstention des salariés des TPE, si massive soit-elle, n'en est pas moins socialement située. Alors que la participation varie fortement d'une branche professionnelle à l'autre, il apparaît que les branches les moins participantes rassemblent un salariat moins âgé, plus précaire et davantage rémunéré au Smic (II.1). Ces résultats au niveau agrégé sont corroborés par les maigres données collectées au niveau individuel et rejoignent ceux obtenus à propos des élections prud'homales et des élections en entreprise (II.2). Dès lors, le caractère massif de l'abstention des salariés des TPE peut aussi s'analyser comme le résultat d'effets structurels, à savoir une précarité plus importante ou encore une ancienneté et une rémunération plus faible de ceux-ci par rapport au reste des salariés du secteur privé.

\section{II.1. Le degré de précarité et l'ancienneté, deux variables explicatives de l'abstention aux scrutins TPE}

Si la participation aux scrutins TPE est globalement faible, on constate, au niveau des branches professionnelles, que le taux de participation est variable. Ainsi, alors que, à l'échelle des branches professionnelles, la participation médiane s'élève à $8,8 \%$ en 2012 et à $7,2 \%$ en 2017 , les écartstypes atteignent respectivement 5,9 et 6,8 points. Or, à l'aide d'analyses multivariées (encadré 2), nous montrons que ces variations sont fortement liées aux caractéristiques du salariat de chaque branche professionnelle.

Comme l'indique le tableau 1, le taux de participation au scrutin TPE est statistiquement lié à l'âge des salariés, à leur rémunération et à leur type de contrat de travail, ces trois variables étant relativement corrélées.

Ainsi, plus la part de salariés de 50 ans ou plus au sein d'une branche est importante, plus la participation au scrutin TPE est importante. Le groupe des branches les plus participantes (groupe 3) rassemble d'ailleurs en moyenne 35,5\% de salariés de 50 ans ou plus contre seulement en moyenne 19,2 \% dans le groupe des branches les moins participantes (groupe 2). De la même manière, plus la part de salariés en CDD au sein 


\section{Présentation des analyses multivariées}

À partir de la base regroupant des données socioéconomiques et électorales pour 231 branches professionnelles (voir supra, encadré 1), nous avons réalisé plusieurs analyses multivariées afin de saisir les liens statistiques entre les caractéristiques du salariat des différentes branches et le taux de participation au scrutin TPE au sein de ces branches.

Tout d'abord, un modèle de régression linéaire a été réalisé avec, en variable dépendante, le taux de participation au sein de la branche. Les variables indépendantes sont la part de salariés à temps partiel, la part de salariés en CDD, la part de salariés rémunérés en dessous de 1,05 Smic, la part de salariés de TPE au sein de la branche (à partir du nombre d'inscrits) ainsi que la part de femmes parmi les salariés, la part de salariés de 29 ans et moins et de 50 ans et plus. Notons toutefois que, si les trois premières variables concernent uniquement les salariés des TPE, les trois dernières portent sur l'ensemble du salariat de la branche. L'ajout d'autres variables, telles que la couverture de la branche par une commission paritaire régionale interprofessionnelle de l'artisanat (CPRIA) ou la représentativité d'un syndicat corporatif autonome dans la branche, n'améliore pas la qualité du modèle (R2 stable à 0,44$)$, et ces variables n'apparaissent pas significatives ${ }^{1}$. Les résultats du modèle sont présentés dans le tableau 1.

Afin de compenser les limites du modèle de régression utilisé, notamment la colinéarité des variables indépendantes, nous avons réalisé une analyse en composante principale (ACP) avec, en variables actives, les sept variables indépendantes du modèle de régression linéaire. Cela nous permet de différencier les branches professionnelles en fonction des caractéristiques sociales de leurs salariés. La participation au scrutin TPE est ensuite projetée en variable supplémentaire. À partir des résultats de l'ACP, non reproduits ici, nous avons pu réaliser une classification ascendante hiérarchique $(\mathrm{CAH})$. Celle-ci fait ressortir trois groupes de branches professionnelles marquées par des caractéristiques et des taux de participation assez différents. La description des groupes est présentée dans le tableau 2.

Le premier groupe rassemble 137 branches de petite taille au sein desquelles les salariés des TPE sont très peu nombreux. Ces branches appartiennent principalement aux secteurs de l'industrie et de la construction. Leur salariat est moins précaire, moins rémunéré au Smic et moins féminisé que dans les deux autres groupes. La participation électorale est proche de la participation moyenne. Le second groupe rassemble 72 branches aux effectifs bien plus importants et appartenant principalement au secteur du commerce (boulangerie, pharmacie, coiffure, immobilier, services de l'automobile...). Les salariés de ces branches sont plus précaires, davantage rémunérés au Smic et plus jeunes que l'ensemble des salariés. La participation au scrutin TPE y est significativement moins importante que dans le reste du salariat. Le troisième groupe rassemble 20 branches marquées par une participation plus importante que la moyenne. II s'agit principalement de branches des services (gardiens d'immeuble, organismes de formation, propreté, cabinets médicaux et dentaires...). Leur salariat est plus âgé, plus féminisé, davantage à temps partiel et plus souvent en dessous de 1,05 Smic que le reste du salariat des TPE. Certaines branches de ce dernier groupe sont marquées par la présence d'un syndicat professionnel non confédéré, comme celle des gardiens d'immeuble ${ }^{2}$ ou des cabinets dentaires ${ }^{3}$, mais cette situation est loin d'être systématique. 
Tableau 1. Résultats du modèle de régression linéaire relatif à la participation au scrutin TPE à l'échelle des branches professionnelles

\begin{tabular}{|l|c|}
\hline Variable & Coefficient \\
\hline Part de salariés à temps partiel & NS \\
\hline Part de salariés en CDD & $-0,06^{*}$ \\
\hline Part de salariés rémunérés à moins de 1,05 Smic & $-0,16^{* * *}$ \\
\hline Part des salariés des TPE dans l'ensemble de la branche & NS \\
\hline Part de salariés de 29 ans et moins & NS \\
\hline Part de salariés de 50 ans et plus & $0,12^{* * *}$ \\
\hline Part de femmes parmi les salariés & $0,07^{* * *}$ \\
\hline R2 ajusté & 0,44 \\
\hline Nombre de branches prises en compte & 231 \\
\hline
\end{tabular}

Note : NS = non significatif ; ${ }^{*}$ : significatif au seuil de $10 \% ;{ }^{* *}$ : significatif au seuil de $1 \%$. Les coefficients du modèle et le $\mathrm{R} 2$ sont arrondis à deux décimales.

Lecture :

- Les coefficients permettent d'estimer le lien entre les différentes variables considérées et le taux de participation au sein de la branche. Plus le coefficient est élevé (en valeur absolue), plus la relation est importante. Des indicateurs de significativité permettent de s'assurer que cette relation n'est pas liée à d'autres variables prises en compte. Enfin, le signe du coefficient (+ ou -) indique le sens de la relation. Ainsi, on peut dire que, après avoir pris en compte l'ensemble des variables mentionnées dans ce tableau, plus la part des salariés en CDD au sein d'une branche augmente, moins la participation électorale au sein de cette branche sera élevée (signe négatif). La relation est toutefois ténue (coefficient de $-0,06$ ) et peu significative (au seuil de $10 \%$ ).

- Le R2 est un indicateur de la qualité du modèle de régression. II représente la part de la variance expliquée par le modèle. II s'élève ici à 0,44 , ce qui signifie que notre modèle explique $44 \%$ de la variance, un résultat assez important.

Source : données DGT, 2013, et Dares, 2012 ; réalisé par l'auteur.

Tableau 2. Description des groupes issus de la classification ascendante hiérarchique

En \% (sauf *)

\begin{tabular}{|c|c|c|c|c|}
\hline & $\begin{array}{c}\text { Groupe } \\
1\end{array}$ & $\begin{array}{c}\text { Groupe } \\
2\end{array}$ & $\begin{array}{c}\text { Groupe } \\
3\end{array}$ & Total \\
\hline Nombre de branches * & 137 & 74 & 20 & 231 \\
\hline Nombre d'inscrits * & 406760 & 1897444 & 229064 & 2533268 \\
\hline Participation moyenne & 10,1 & 8,4 & 12,3 & 9,8 \\
\hline $\begin{array}{l}\text { Part moyenne des salariés des TPE } \\
\text { dans l'ensemble de la branche }\end{array}$ & 15,8 & 59,5 & 30,0 & 29,8 \\
\hline Part moyenne de salariés de 29 ans et moins & 15,8 & 30,3 & 14,5 & 20,3 \\
\hline Part moyenne de salariés de 50 ans et plus & 28,2 & 19,2 & 35,5 & 26,0 \\
\hline Part moyenne de femmes salariées & 28,1 & 51,7 & 70,2 & 39,3 \\
\hline Part moyenne de salariés à temps partiel & 17,4 & 26,9 & 48,6 & 23,2 \\
\hline Part moyenne de salariés en CDD & 6,8 & 11,2 & 9,7 & 8,4 \\
\hline $\begin{array}{l}\text { Part moyenne de salariés rémunérés } \\
\text { à moins de } 1,05 \mathrm{Smic}\end{array}$ & 6,2 & 13,5 & 15,0 & 9,3 \\
\hline
\end{tabular}

Source : données DGT, 2013, et Dares, 2012 ; ACP et CAH réalisées avec FactoMineR.

1. Notons que, parmi les 231 branches pour lesquelles nous disposons de données, moins de $5 \%$ sont entièrement couvertes par une CPRIA ou se caractérisent par la représentativité d'un syndicat professionnel non confédéré.

2. Avec la présence du Syndicat national indépendant des gardiens d'immeubles et concierges (Snigic). 3. Avec la présence de la Fédération nationale indépendante des syndicats des prothésistes et assistants(es) dentaires (Fnispad). 
d'une branche est importante, plus le taux de participation y est faible. Si cette relation est ténue (significative au seuil de $10 \%$ ), le groupe des branches les moins participantes (groupe 2) est marquée par un recours plus important aux CDD (en moyenne 11,2 \% dans le groupe 2 contre en moyenne 8,4\% dans l'ensemble des branches) (tableau 2).

Le tableau 1 indique en outre que plus les branches ont un salariat ayant une rémunération inférieure à 1,05 Smic, moins celles-ci sont participantes. Cette relation est d'ailleurs la plus significative et la plus conséquente. Toutefois, si les branches les moins participantes (groupe 2) sont marquées par une surreprésentation des salariés ayant une rémunération inférieure à 1,05 Smic (13,5\% dans le groupe 2 contre en moyenne 9,3\% dans l'ensemble du salariat), c'est aussi le cas des branches les plus participantes (groupe 3) (en moyenne $15 \%$ ). Enfin, un lien entre la participation et la féminisation des branches apparaît dans le tableau 1. Plus les branches sont féminisées, plus la participation y est importante. Mais, là encore, les résultats présentés dans le tableau 2 nous invitent à relativiser cette corrélation. Ainsi, si les branches les plus participantes (groupe 3) sont très féminisées (en moyenne 70,2 \% contre en moyenne 39,3\% dans l'ensemble des branches), c'est aussi le cas des branches les moins participantes (groupe 2) (en moyenne 51,7\%).

\section{II.2. Des résultats convergents avec ceux obtenus au niveau individuel et pour d'autres scrutins professionnels}

Les résultats au niveau agrégé que nous avons présentés précédemment semblent se confirmer au niveau individuel. Ainsi, à l'image de ce qui a été observé dans le tableau 1, les maigres données issues de notre enquête par questionnaire tendent à montrer que le fait d'être à temps partiel joue peu (Chi-Deux non significatif) ${ }^{12}$. À l'inverse, comme le suggèrent les résultats des analyses multivariées, le type de contrat, le genre et l'ancienneté des salariés semblent influer sur la participation (Chi-Deux significatifs au seuil de $5 \%$ ). Les femmes salariées déclarent moins s'être abstenues que les hommes salariés. De plus, les salariés en CDD ou ayant un autre type de contrat précaire déclarent significativement moins avoir participé aux scrutins TPE que les salariés en CDI ${ }^{13}$. Enfin, plus un salarié est âgé et ancien dans son emploi, plus il a de chances d'avoir voté aux scrutins TPE. Il pourrait s'agir du résultat d'un « effet de génération », lié à la socialisation et aux opinions des salariés les plus jeunes, ou d'un « effet d'âge », lié à l'instabilité professionnelle qu'implique leur jeune âge (Béroud et al., 2018, 2019 ; Chartier, 2018).

12. Lorsque le résultat du test du Chi-Deux n'est pas significatif, cela signifie que la répartition des réponses selon la variable étudiée peut être due au hasard.

13. À ce titre, rappelons que les femmes salariées des TPE sont légèrement moins en CDD que les hommes (Chausse, 2020). 
Ces résultats convergent également avec ceux observés lors d'autres scrutins professionnels. Un certain nombre de travaux ont en effet porté sur la participation aux scrutins prud'homaux (Blanchard et al., 1996 ; Subileau, 1999) et, plus récemment, sur la participation aux élections de représentants du personnel en entreprise (Haute, 2019a) et dans la fonction publique (Haute, 2019b). À partir des enquêtes REPONSE et SRCV, nous avons montré dans notre thèse que les salariés les plus précaires, en CDD, en intérim, voire à temps partiel, votent moins aux élections professionnelles en entreprise et y sont moins confrontés (Haute, 2019a:228-250). Par ailleurs, ces salariés votaient déjà significativement moins aux scrutins prud'homaux (Subileau, 1999:164) et ils s'abstiennent significativement plus aux scrutins politiques (Peugny, 2015). De la même manière, les salariés les plus jeunes sont victimes d'une forme de «moratoire électoral»: tout en n'étant pas plus critiques à l'égard du vote et des syndicats, ils sont significativement moins syndiqués (Béroud et al., 2019:7-9), se confrontent moins à l'employeur (Trémeau, 2019a) et participent moins aux scrutins professionnels (Blanchard et al., 1996:48 ; Haute, 2019a:265 ; Haute, 2019b:152) ou politiques, notamment quand ceux-ci sont peu mobilisateurs (Braconnier et al., 2017:1037) ${ }^{14}$. Enfin, la participation électorale plus importante des salariées par rapport aux salariés lors des scrutins TPE peut certes paraitre contradictoire avec la plus faible syndicalisation de celles-ci (Lescurieux, 2019:96), avec leur moindre propension à se confronter à leur employeur (Trémeau, 2019a) et avec un intérêt plus faible pour la politique (Achin, Lévêque, 2018:38). On peut toutefois souligner que ce résultat était déjà observé lors des scrutins prud'homaux (Blanchard et al., 1996:53) et qu'on le retrouve tant aux élections professionnelles en entreprise (Haute, 2019a:268) que dans le champ politique (Tiberj, 2018:831) ${ }^{15}$. De plus, les femmes salariées des TPE ont une opinion plus positive des syndicats que les hommes : selon une enquête d'opinion réalisée pour la CGT, $66 \%$ d'entre elles estiment que «les syndicats permettent aux salariés de trouver des lieux d'écoute et de conseil partout sur le territoire » contre $56 \%$ des hommes salariés des TPE ${ }^{16}$. Cette énigme du genre pourrait s'expliquer par un rapport genré à la politique et à la protection sociale construit dès l'adolescence dans le cadre du processus de socialisation (Achin, Lévêque, 2018:42).

Lors des scrutins TPE, la participation des salariés, si faible soit-elle, n'en est pas moins socialement située. La rémunération, le degré de précarité ou l'ancienneté constituent autant de variables « lourdes » de la participation

14. Des résultats similaires ont été obtenus s'agissant des élections syndicales en Espagne (Malo, 2006).

15. Mais pas dans la fonction publique (Haute, 2019b:151).

16. Enquête réalisée en ligne par Harris Interactive du 20 au 30 juillet 2012 auprès d'un échantillon de 1039 répondants représentatif des salariés des TPE et établi selon la méthode des quotas : https://bit.ly/39RC4ab. 
électorale, à l'image de ce qui a par ailleurs été observé en entreprise. Or, les salariés des TPE se distinguent du reste du salariat par d'importantes spécificités socioéconomiques. Ils sont ainsi plus précaires, plus jeunes et moins rémunérés que les salariés des autres entreprises (Farvaque, Lefebvre, 2010:9). Tout d'abord, les TPE recourent, plus fréquemment que d'autres entreprises, au temps partiel et aux CDD. En 2018, 27,5 \% des salariés des TPE sont à temps partiel contre 17,6\% dans les autres entreprises et 13,2\% des salariés des TPE sont en CDD contre 9,9\% dans les entreprises de 10 à 19 salariés (Chausse, 2020). En outre, le salaire mensuel net des salariés des TPE (1 810 euros en 2012) est nettement inférieur au salaire net moyen de l'ensemble du salariat du secteur privé (2 170 euros en 2012). Enfin, toujours en 2012, la part des salariés rémunérés à moins de 1,05 Smic atteint 12,7 \% dans les TPE contre 7,1 \% dans l'ensemble du secteur privé.

Dès lors, à la lumière des résultats précédemment présentés, la très faible participation des salariés des TPE pourrait être en partie la conséquence d'un effet structurel, à savoir des conditions d'emploi particulièrement précaires et une faible rémunération et une moindre intégration professionnelle par rapport aux salariés des autres entreprises. Cependant, ces spécificités salariales ne peuvent, à elles seules, expliquer la faible participation des salariés des TPE. Une autre différence fondamentale entre les salariés des TPE et le reste des salariés doit être abordée : les salariés des TPE sont en effet plus atomisés et moins confrontés à l'action syndicale que les autres salariés.

\section{Une abstention massive qui résulte d'une distance entre les salariés des TPE et les syndicats}

Les salariés des TPE se distinguent des autres salariés par leur atomisation et par leur distance pratique aux syndicats, ces derniers étant très peu implantés dans les TPE, hormis dans quelques branches professionnelles spécifiques, et éprouvant des difficultés pour s'adresser aux salariés des TPE (III.1). Or, les contacts entre les salariés et les syndicats, qui peuvent prendre des formes variables, sont parmi les principaux facteurs de la mobilisation électorale (III.2).

\section{III.1. Des salariés atomisés et à distance des syndicats}

Faute de collectif de travail ${ }^{17}$ ou du fait de collectifs de travail réduits ou instables, le « dialogue social» prend, dans les TPE, une tournure individuelle, informelle et «domestique ». Les relations sociales dans les TPE

17. Rappelons que la forme monosalariale concernait $12,6 \%$ des salariés des TPE en 2016 (Le-Ninivin, 2018). 
sont avant tout faites d'arrangements individuels non formalisés et de contournements du droit du travail, y compris en matière de rémunération, ce qui limite l'intérêt de l'adhésion syndicale, de la conflictualité collective et de la représentation collective des salariés ${ }^{18}$. De plus, l'homologie sociale relative entre les salariés et les employeurs, notamment dans l'artisanat (Gassier, 2020; Mazaud, 2012), et la persistance de logiques de métier (Trémeau, 2019a) conduisent à occulter le rapport asymétrique entre les salariés et les employeurs et renforce le pouvoir de coercition de ces derniers. Or, une grande partie des employeurs sont traditionnellement opposés à l'existence de droits syndicaux dans les TPE et à toute représentation collective des salariés, y compris hors de l'entreprise ${ }^{19}$. De ces éléments découle une implantation syndicale extrêmement rare dans les TPE ainsi que dans les secteurs d'activité où les TPE sont majoritaires, comme le commerce, le bâtiment, les services à la personne ou le secteur associatif (Trémeau, 2019b:64).

Il existe dès lors une forme de distance pratique entre les syndicats et les salariés des TPE, comme l'illustrent les difficultés des premiers pour s'adresser aux seconds (encadré 3). Les rares salariés de TPE qui rencontrent les syndicats cherchent auprès d'eux un appui juridique, en sollicitant une structure territoriale ou un conseiller du salarié dans le cadre d'un litige avec l'employeur qui se règle aux prud'hommes (Trémeau, 2019b:80 ; Sin, Grima, 2019:66-67). Cette distance pratique implique une faible information des salariés sur les scrutins TPE et une faible réception des campagnes syndicales, à l'image de ce qui était observé lors des élections prud'homales (Blanchard et al., 1996:73-74 ; Farvaque, Lefebvre, 2010:29-30).

Cette distance structurelle ne se traduit toutefois pas par des opinions plus négatives et défiantes à l'égard des syndicats. Selon l'enquête d'opinion citée précédemment, si $64 \%$ des salariés des TPE estiment que «les syndicats sont souvent un facteur de problèmes en entreprise », ils sont $62 \%$ à considérer que « les syndicats fournissent une assistance indispensable aux salariés ». De même, $60 \%$ des répondants estiment que «les syndicats permettent aux salariés de trouver des lieux d'écoute et de conseil partout sur le territoire » et ils sont même $47 \%$ à déclarer être prêts à adhérer à un syndicat " pour défendre leurs intérêts et ceux de leurs collègues ». En d'autres termes, l'éloignement des salariés des TPE vis-à-vis des syndicats s'explique moins par leurs opinions que par des facteurs structurels propres aux TPE, à l'image de la moindre syndicalisation des jeunes qui

18. Nous renvoyons ici à un article récent de Céline Dumoulin mobilisant les données de l'enquête REPONSE et portant sur les établissements de 11 à 49 salariés (Dumoulin, 2019).

19. Même si rares sont les employeurs (entre 9 et $10 \%$ ) à recourir aux services d'une organisation patronale (Desage, Rosankis, 2016 ; Tall, 2018), les deux principales organisations d'employeurs (Mouvement des entreprises de France, Medef ; Confédération générale des petites et moyennes entreprises, CGPME) se sont toujours opposées à la mise en place des CPRI. 
s'explique bien plus par un « effet d'âge » lié à l'instabilité et à la précarité que subissent les salariés les plus jeunes qu’à un « effet de génération » lié à des opinions plus critiques à l'égard des syndicats (Béroud et al., 2018, 2019 ; Chartier, 2018).

\section{Encadré 3}

\section{Les stratégies d'accès aux salariés lors des campagnes TPE}

Durant les campagnes TPE, les syndicats ont éprouvé des difficultés pour accéder aux salariés des TPE.

Une première stratégie d'accès aux salariés des TPE consiste à mener des " opérations de rencontres » avec des salariés au sein de leur entreprise. Mais les organisations syndicales peinent à recenser les TPE, à y entrer et à y distinguer les salariés de l'employeur ${ }^{1}$. Le recours à cette stratégie est donc très inégal ${ }^{2}$.

Les syndicats construisent dès lors une relation avec les salariés par voie épistolaire ou numérique. La « proximité » revendiquée se joue paradoxalement à distance. Les syndicats envoient du matériel syndical en utilisant la liste électorale diffusée par le ministère du Travail qui contient les adresses des électeurs. Ces envois constituent l'activité majeure de la campagne TPE pour nombre de nos interlocuteurs syndicaux et cela malgré les nombreuses erreurs d'inscription sur la liste électorale (voir supra, I.1). Les envois, gérés nationalement ou au niveau territorial, sont parfois ciblés, au sens où le matériel envoyé aux salariés est spécifique à leur branche professionnelle. Certaines organisations comme I'UNSA recourent également au démarchage par téléphone ou par courriel, mais ces dispositifs de mobilisation demeurent coûteux.

En parallèle de cette campagne de proximité contrainte, des dispositifs de mobilisation à destination d'un public plus large ont été mis en place à divers niveaux (réunions publiques, affichages, présence dans des lieux de passage...). Leur nombre et leurs formes varient d'une organisation à l'autre et les déclinaisons locales des dispositifs nationaux sont très inégales. De plus, selon les syndicalistes enquêtés, l'efficacité de tels dispositifs est très discutée en interne, tant le nombre de salariés des TPE rencontrés par ce biais semble faible.

1. Certaines organisations comme l'UNSA assument de s'adresser aussi aux employeurs et les invitent à faire participer leurs salariés (entretien de l'auteur avec un responsable territorial de I'UNSA, 9 décembre 2017).

2. Pour un état des lieux du recours aux différents dispositifs de mobilisation par chaque organisation syndicale durant les campagnes TPE, voir Haute (2019a:192-201).

Dans ce contexte, des syndicats corporatifs autonomes développent exclusivement des actions de représentation et d'appui juridique et bénéficient dès lors de la bienveillance des employeurs. C'est le cas notamment dans le secteur sportif (Falcoz, Lefèvre, 2016:12-14), parmi les gardiens d'immeubles, les assistants dentaires ou les assistantes maternelles (encadré 4). Ces organisations syndicales professionnelles corporatives sont 


\section{Encadré 4}

\section{L'exemple des assistantes maternelles}

Nous souhaitons ici nous arrêter sur le cas des assistantes maternelles. Ces salariées ${ }^{1}$, essentiellement en CDI et à temps plein mais très faiblement rémunérées, représentent un peu plus de $6 \%$ des inscrits et $14 \%$ des votants aux scrutins TPE. Dans la branche des assistantes maternelles, la participation au scrutin TPE a atteint 16,1\% en 2017 contre 7,3 \% dans l'ensemble du salariat des TPE.

Les deux organisations syndicales récoltant le plus de suffrages dans cette branche professionnelle sont deux syndicats autonomes non confédérés : le Syndicat professionnel des assistants maternels et assistants familiaux (SPAMAF) et la Confédération des syndicats d'assistants familiaux et d'assistants maternels (CSAFAM). La troisième organisation représentative dans la branche, I'UNSA, est également issue d'un syndicat autonome, le Syndicat d'union professionnel national - assistants/accueillants familiaux et assistants maternels (SUPNAAFAM). Ces différentes structures syndicales sont issues d'associations professionnelles anciennes qui ont progressivement pris le statut de syndicats. Si la structuration de la représentation des assistantes maternelles était bien antérieure aux scrutins TPE, la représentation des employeurs l'était également avec la création de la Fédération des particuliers-employeurs (FéPEm) dès 1948. La branche des assistantes maternelles connaît enfin une activité intense en matière de négociation collective, notamment par rapport à d'autres conventions au sein desquelles les salariés des TPE sont majoritaires.

Les organisations corporatives d'assistantes maternelles offrent avant tout un appui juridique et administratif aux assistantes maternelles alors que leur convention collective est très peu protectrice. En effet, si la conflictualité gréviste est inexistante du fait du caractère monosalarial de la TPE, le recours aux prud'hommes n'est pas rare (Chavaroche-Laurent, 2012:30). De plus, la syndicalisation n'est pas forcément mal perçue par la multitude des employeurs. Les parents employeurs se considèrent en effet rarement comme tels. Les assistantes maternelles entretiennent une certaine proximité avec ces derniers et les parents les laissent souvent rédiger elles-mêmes leur contrat de travail et calculer leur salaire, l'appui administratif du syndicat étant ici particulièrement utile (Chavaroche-Laurent, 2012:29).

1. $1 \%$ des demandes d'agrément sont faites par des hommes.

mieux implantées que les syndicats interprofessionnels, du moins au sein des groupes professionnels qu'elles représentent, et elles parviennent davantage à mobiliser électoralement les salariés lors des scrutins TPE. Les audiences de ces organisations, si elles se limitent souvent à une seule convention collective, sont non négligeables : ces organisations ont ainsi obtenu 10,5\% des voix au scrutin de 2012 à l'échelle interprofessionnelle et $10,3 \%$ des voix au scrutin de 2017 (voir infra, encadré 5, tableau 3). À 
titre de comparaison, ces mêmes organisations ont obtenu, lors des scrutins en entreprise, moins de $3 \%$ des voix entre 2009 et 2016.

Hormis dans ces cas très spécifiques, les salariés des TPE sont à distance de syndicats qui peinent à s'adresser à eux et à les informer, y compris lors des scrutins TPE. C'est là le principal élément explicatif de l'abstention massive enregistrée lors des scrutins TPE puisque ce sont les interactions entre les salariés et les syndicats qui sont les vecteurs les plus efficaces de la participation électorale.

\section{III.2. Les contacts avec les syndicats, principaux vecteurs de la mobilisation électorale}

Les contacts, même rares, que nouent les salariés des TPE avec les syndicats sont les principaux facteurs explicatifs de leur participation ou non aux scrutins TPE.

Ainsi, selon les données de notre enquête par questionnaire, la lecture d'informations syndicales, le fait de savoir que ses collègues votent ou encore le fait d'avoir des discussions relatives au syndicalisme avec des collègues vont de pair avec une participation électorale plus importante (Chi-Deux significatifs au seuil de $5 \%$ ), un résultat conforme à ceux obtenus s'agissant des élections en entreprise (Haute, 2019a:317-323). Si les discussions avec des amis à propos du syndicalisme jouent moins (Chi-Deux non significatif), il convient toutefois de ne pas négliger les influences extérieures à l'entreprise, le vote ne se déroulant pas sur le lieu de travail mais à domicile. Camille Trémeau a d'ailleurs bien montré que la socialisation familiale façonnait les attitudes et représentations des jeunes salariés à l'égard du syndicalisme (Trémeau, 2019b:73).

Les contacts, formels ou informels, qu'entretiennent les salariés avec les syndicats permettent l'activation de prédispositions ou la « conversion » de salariés à la pratique électorale. Comme cela avait été observé lors des scrutins prud'homaux, la connaissance d'un militant ou d'un candidat permet de rendre le syndicalisme moins opaque et le vote moins improbable (Farvaque, Lefebvre, 2010:30). Cependant, si les militants syndicaux et les représentants du personnel sont, en entreprise, autant de «leaders d'opinion » qui encouragent la participation électorale de leurs collègues (Haute, 2019a:311-316), l'absence de représentants et le faible nombre de militants syndicaux dans les TPE impliquent dès lors que ces effets d'entraînement sont bien moins nombreux lors des scrutins TPE. Ils concernent toutefois deux populations distinctes.

D’un côté, on trouve des salariés qui sont non seulement politisés et qui manifestent des opinions positives à l'égard du syndicalisme, mais aussi qui participent politiquement dans et hors du travail. On peut citer l'exemple de cet animateur de 30 ans dans le secteur associatif, diplômé de l'enseignement supérieur (bac +4 ou plus) et se positionnant très à gauche. Il déclare 
lire les informations syndicales, discuter de l'action des syndicats avec des amis et avoir participé à plusieurs actions collectives et à une grève. Il a eu connaissance du scrutin par l'intermédiaire de tracts et d'affiches. Il a voté en faveur de la Confédération nationale du travail (CNT), du fait des valeurs portées par ce syndicat. À l'inverse, on peut avancer le cas de ce développeur en informatique, diplômé de l'enseignement supérieur (bac+4 ou plus), se positionnant à gauche et au fait du scrutin par l'intermédiaire de tracts. Celui-ci n’a pourtant pas voté, considérant que cela était inutile, ce qui légitime par ailleurs les explications de l'abstention par l'absence d'enjeu (voir supra, I.2). Or, ce salarié ne déclare aucun lien avec les syndicats, tout en ayant une opinion positive de ceux-ci.

D'un autre côté, les effets d'entrainement décrits plus haut concernent également des salariés bien moins politisés et mobilisés, mais appartenant à des groupes professionnels fortement structurés par un syndicalisme corporatif de services, comme l'illustrent les résultats de la classification présentés plus haut (voir supra, tableau 2), et notamment la composition du troisième groupe (voir supra, encadré 2). Le cas des assistantes maternelles est également exemplaire (voir supra, encadré 4) et certaines d'entre elles expliquent d'ailleurs leur participation par la rencontre avec des militants (15 répondantes sur 47) ou par des discussions avec des collègues ou amis (six répondantes). Au sein de ces groupes, le vote est moins l'expression d'opinions ou de préférences individuelles qu'un «acte de conformité sociale symbolisant l'appartenance au groupe » (Gaxie, 1978:224 ; Lehingue, 2011:77). Le vote revêt dès lors une dimension symbolique et rituelle au sens où il constitue, pour des personnes au statut marginalisé, un moyen de renforcer leur lien avec le groupe professionnel de référence (Lehingue, 2011:78). Dans les faits, le vote est alors souvent un prolongement de l'adhésion à un syndicat corporatif, cette dernière étant l'une des conditions d'une bonne intégration professionnelle. Les données de notre enquête par questionnaire confirment d'ailleurs que, pour les assistantes maternelles, la mobilisation électorale n'est pas corrélée à la participation à des actions collectives. Ce résultat explique sans doute le moindre recul, en nombre de voix, des syndicats professionnels non confédérés entre 2012 et 2017 (encadré 5).

Pour terminer, la connaissance de militants en dehors des cadres professionnels ou syndicaux peut aussi, en soi, permettre la participation de salariés peu politisés et mobilisés. C'est ce qu'illustre le cas de ce technicien de 28 ans, diplômé de l'enseignement supérieur (bac +4 ou plus). Il déclare ne pas avoir participé à des actions collectives, ne pas avoir de contact avec les syndicats et se positionner au centre sur un axe gauche-droite. Cependant, et alors qu'il développe une conception peu conflictuelle du syndicalisme, il a voté pour l'union syndicale Solidaires. Il justifie ce choix improbable par la rencontre avec des militants de ce syndicat dans le cadre de ses loisirs. 


\section{Encadré 5}

\section{Retour sur les résultats des scrutins TPE de 2012 et de 2017}

Si les scrutins TPE ont été marqués par une très faible participation en 2012 et en 2017 , la forte baisse de cette même participation entre les deux scrutins nous invite à étudier les évolutions des résultats syndicaux. À ce titre, une lecture de ces résultats en nombre de voix permet de tenir compte de la baisse de la participation.

Le tableau 3 montre ainsi que, en 2017, il y avait près d'un tiers de votants de moins qu'en 2012. Les principales organisations syndicales confédérées (CGT, CFDT, FO) ainsi que Solidaires et la Confédération autonome du travail (CAT) ont perdu en 2017 plus d'un tiers et parfois près de la moitié des suffrages obtenus en 2012, alors que la CFTC, la Confédération nationale du travail (CNT) et les syndicats professionnels non confédérés ou ultramarins ont enregistré des reculs moins importants en nombre de voix ${ }^{1}$. La CFECGC voit au contraire son nombre de voix légèrement augmenter, ce qui n'est pas surprenant au regard de l'augmentation, entre les deux scrutins, du poids des cadres parmi les inscrits et parmi les votants. Enfin, I'UNSA progresse tant en pourcentage $(+5,1$ points) qu'en nombre de votes avec un peu moins de 6500 voix en plus entre 2012 et 2017.

Tableau 3. Résultats des scrutins TPE de décembre 2012 et de janvier 2017 En nombre et en \%

\begin{tabular}{|l|r|r|r|r|}
\hline \multicolumn{3}{|c|}{ Voix } & En \% & \multicolumn{2}{c|}{ Voix } & En \% \\
\hline Inscrits & 4614653 & & 4502621 & \\
\hline Votants & 478866 & 10,4 & 330928 & 7,3 \\
\hline Exprimés & 465796 & & 323622 & \\
\hline CGT & 136033 & 29,5 & 81286 & 25,1 \\
\hline CFDT & 88699 & 19,3 & 50122 & 15,5 \\
\hline FO & 70231 & 15,3 & 42117 & 13,0 \\
\hline UNSA & 33864 & 7,4 & 40429 & 12,5 \\
\hline CFTC & 30074 & 6,5 & 24082 & 7,4 \\
\hline Solidaires & 21885 & 4,8 & 11324 & 3,5 \\
\hline CFE-CGC & 10697 & 2,3 & 10928 & 3,4 \\
\hline CNT-F & 9818 & 2,2 & 7436 & 2,3 \\
\hline CNT-SO & & & 5844 & 1,8 \\
\hline SAP & 10995 & 2,4 & 6790 & 2,1 \\
\hline CAT & 775 & 0,2 & 3899 & 1,2 \\
\hline STC & 48152 & 10,5 & 33472 & 1,1 \\
\hline Autres (hors ultramarins) & 4573 & 0,9 & 2405 & 10,3 \\
\hline Outre-mer & & & & 0,7 \\
\hline
\end{tabular}

Note : les chiffres sont arrondis à une décimale. Plus précisément, le scrutin de 2012 a eu lieu entre le 28 novembre 2012 et le 12 décembre 2012 et le scrutin de 2017 s'est déroulé entre le 30 décembre 2016 et le 13 janvier 2017. Le Syndicat des travailleurs corses (STC) n'était candidat en 2012 que sur le périmètre régional de Corse. En 2017, il était candidat sur tout le territoire français, ce qui est le résultat du contentieux juridique qui a par ailleurs conduit au report du scrutin de plusieurs semaines. Ce changement de périmètre explique en partie la progression du syndicat. Source : données DGT, 2013 et 2017. 
Cette lecture en nombre de voix à l'échelle interprofessionnelle masque toutefois le poids de certaines branches professionnelles. Ainsi, près des trois quarts des suffrages en faveur des " autres syndicats non confédérés " se sont dans les faits portés sur les organisations professionnelles d'assistantes maternelles ( $70 \%$ pour le SPAMAF en $2012,73 \%$ pour le SPAMAF et la CSAFAM en 2017). De la même manière, plus de $30 \%$ des voix supplémentaires obtenues par l'UNSA en 2017 (un peu moins de 2000 sur un peu moins de 6500 ) sont le résultat d'une progression importante de l'UNSA parmi les assistantes maternelles avec le développement du SUPNAAFAM devenu UNSA ProAssMat.

1. La CNT progresse si on ajoute les voix des deux CNT en 2017. En effet, la CNT-SO (Solidarité ouvrière), qui ne s'est présentée qu'en 2017, est le résultat d'une scission de la CNT dite « $F$ ».

Nos maigres données recueillies au niveau individuel tendent à montrer que les votants ne se distinguent pas des abstentionnistes par un recours à la grève plus important, par une image du syndicalisme plus positive ou par une conception de ce dernier plus « revendicative» ou plus « pragmatique ». On note ainsi que les salariés les plus jeunes, que les salariés les moins bien rémunérés et que les salariés en CDD participent moins que le reste des salariés mais ont, selon l'enquête d'opinion citée précédemment, des opinions plus positives à l'égard du syndicalisme ${ }^{20}$. Les données d'enquête donnent à voir, au contraire, des rapports des salariés des TPE au syndicalisme très variés et qui ne peuvent se résumer à la défiance ou à l'indifférence. À l'image de ce qui est observé en matière de rapports au syndicalisme des jeunes salariés, on assiste à un hiatus entre pratiques syndicales et opinions à l'égard du syndicalisme (Trémeau, 2019b:83-84).

Si l'abstention s'explique par un éloignement des syndicats, celui-ci est plus physique que théorique, les organisations syndicales éprouvant de réelles difficultés pratiques à toucher les salariés des TPE. Les rares contacts directs avec le syndicalisme, s'ils peuvent être insuffisants, constituent d'ailleurs les stimuli électoraux les plus efficaces. Dès lors, l'abstention de l'écrasante majorité des salariés aux scrutins TPE s'explique en grande partie par le caractère résiduel de l'implantation syndicale dans les TPE et par la faiblesse des contacts entre les syndicats et les salariés des TPE. Si certaines branches connaissent une participation plus importante, mais toujours relativement faible en comparaison avec les taux de participation aux élections de représentants du personnel en entreprise, c'est grâce à l'implantation de syndicats confédérés ou non développant une action corporative et construisant le vote comme un acte rituel d'appartenance au groupe professionnel qu'ils représentent.

20. Les salariés du particulier employeur, parmi lesquels on trouve les assistantes maternelles, semblent cependant avoir des opinions plus positives que les salariés de l'artisanat notamment. 


\section{Conclusion}

La participation aux scrutins TPE a été résiduelle en 2012 et en 2017. Cette abstention massive est moins le résultat d'une défiance des salariés des TPE à l'égard des syndicats que la conséquence de facteurs structurels. L'abstention s'explique non seulement par les spécificités des scrutins TPE, à savoir des conditions de déroulement du vote peu favorables à la mobilisation et une absence d'enjeu apparent, mais aussi par les spécificités du salariat des TPE. D’une part, alors que les salariés les plus précaires, les moins bien rémunérés et les moins intégrés professionnellement s'abstiennent davantage que l'ensemble des salariés, la faible participation des salariés des TPE est à mettre en lien avec leur plus grande précarité et avec leur moindre rémunération par rapport au reste des salariés. D'autre part, puisque les contacts entre les syndicats et les salariés des TPE sont les principaux vecteurs de la mobilisation électorale, l'abstention massive enregistrée lors des scrutins TPE s'explique par la distance pratique entre les salariés des TPE et les syndicats. Ces derniers sont peu implantés dans les TPE et parviennent difficilement à s'adresser et à mobiliser les salariés des TPE, hormis dans quelques branches professionnelles, comme celle des assistantes maternelles, où domine un modèle syndical serviciel. Ces différentes logiques de l'abstention, loin d'être contradictoires, s'imbriquent et peuvent se cumuler. Ainsi, l'implantation syndicale et la prise de contact avec les salariés sont d'autant plus aisés que la main-d'œuvre d'un secteur d'activité est stable.

Ces premières pistes conclusives nécessitent d'être mises à l'épreuve de données plus robustes, notamment au niveau individuel. Le scrutin de 2021 pourrait représenter une occasion de produire de telles données, même si des évolutions importantes sont envisagées. Ces évolutions ne devraient pas modifier les facteurs structurels exposés dans cet article. Néanmoins, il est à noter que, pour être candidates, les organisations syndicales devront déposer des listes paritaires de candidats salariés des TPE (10 par région), ces salariés étant amenés à siéger dans les CPRI. Cette nouvelle nécessité pourrait certes diminuer l'offre syndicale, mais elle contraint les organisations syndicales à recruter des adhérents et à former des militants issus des TPE. De même, le vote, qui se déroulait jusqu'ici par correspondance ou en ligne, pourrait se dérouler exclusivement par Internet, ce qui risque d'engendrer une nouvelle baisse de la participation. Il sera dès lors intéressant de comparer la participation des salariés à ce nouveau scrutin avec les résultats des analyses présentées dans cet article afin d'estimer, dans le champ professionnel, l'impact de ces deux évolutions électorales. 


\section{Références bibliographiques}

Achin C., Lévêque S. (2018), " Mind the Gap ! De la variable sexe au genre des comportements électoraux ॥, Travail, genre et sociétés, $n^{\circ} 40$, p. 33-50, https://doi.org/10.3917/tgs.040.0033.

Andolfatto D. (2017), « Réformer ou inventer une démocratie sociale "à la française" : innovation ou échec ? ", $7^{\mathrm{e}}$ congrès de l'Association belge de science politique (ABSP), Mons, 3 avril, https://hal.archives-ouvertes.fr/ hal-01698169.

Béroud S., Chartier F., Dupuy C., Kahmann M., Yon K. (2018), Jeunes et mouvement syndical. Trajectoires d'engagements et stratégies organisationnelles, Rapport réalisé pour la CGT dans le cadre de l'agence d'objectifs de l'Ires, janvier, https://goo.gl/gGXNz2.

Béroud S., Dompnier N. (2013), « L'essor du vote électronique dans les élections professionnelles : un terrain d'expérimentation ? », Droit social, n 6, p. 522528, https://halshs.archives-ouvertes.fr/halshs-00859524.

Béroud S., Dupuy C., Kahmann M., Yon K. (2019), « Jeunes et engagements au travail : une génération asyndicale ? ", Agora débats/jeunesses, n 82, p. 7-25, https://doi.org/10.3917/agora.082.0007.

Béroud S., Guillaume C. (2013), « La campagne TPE : une opportunité de réflexion sur les pratiques syndicales ? ", in Béroud S., Yon K., Denis J.-M., Gantois M., Guillaume C. (dir.), Quand la loi entre dans les moeurs. Le nouveau droit de la représentativité syndicale (loi du 20 août 2008) et ses implications dans les entreprises et les branches professionnelles, Rapport final pour la Dares, mars, p. 224-245, https://hal.archives-ouvertes. fr/hal-00830449/document.

Blanchard M., Bouhet B., Geoffroy M., Mouriaux R., Subileau F. (1996), L'abstention aux élections prud'homales, Rapport final.

Braconnier C., Coulmont B., Dormagen J.-Y. (2017), « Toujours pas de chrysanthèmes pour les variables lourdes de la participation électorale : chute de la participation et augmentation des inégalites électorales au printemps 2017 », Revue française de science politique, $n^{\circ}$ 67, p. 1023-1040, https://doi.org/10.3917/rfsp.676.1023.

Chartier F. (2018), "Pourquoi les jeunes salariés sont-ils moins syndiqués ? ", Éclairages, $n^{\circ} 010$, Ires, mai, https://bit.ly/2yz4XL4.

Chausse M.-L. (2020), « L'emploi dans les très petites entreprises fin 2018 », Dares Résultats, $\mathrm{n}^{\circ}$ 004, février, https://dares.travail-emploi.gouv.fr/lMG/pdf/ dares_resultats_emploi_tpe_2018.pdf.

Chavaroche-Laurent M.-C. (2012), « Une profession invisible, des salariées invisibles : les assistantes maternelles $"$, Vie sociale et traitements, $n^{\circ} 116$, p. 27-30, https://doi.org/10.3917/vst.116.0027.

Desage G., Rosankis E. (2016), « Le dialogue social dans les très petites entreprises », Dares Résultats, $\mathrm{n}^{\circ}$ 062, novembre, https://dares.travail-emploi. gouv.fr/IMG/pdf/2016-062.pdf.

Dompnier N. (2018), « Le "bilan largement positif" des élections de 1987 : enjeux politiques de l'organisation et de la contestation d'un scrutin prud'homal », in Le Digol C., Hollard V., Voilliot C., Barat R. (dir.), Histoires d'élections. Représentations et usages du vote de l'Antiquité à nos jours, Paris, CNRS Éditions, p. 457-475, https://halshs.archives-ouvertes.fr/halshs-02097559. 
Dumoulin C. (2019), «Quand les syndicats s'invitent dans les petites entreprises. Les relations sociales dans les établissements de 11 à 49 salariés », La Nouvelle Revue duTravail, n 15, https://doi.org/10.4000/nrt.5817.

Falcoz M., Lefèvre N. (2016), « Représenter les sportifs professionnels : entre militantisme apolitique et syndicalisme de service ", Staps, $n^{\circ} 113$, p. 7-20, https://doi.org/10.3917/sta.113.0007.

Farvaque N., Lefebvre M. (2010), « Les salariés de PME : d'une spécificité des relations sociales à un rapport particulier aux Prud'hommes ? ", La Revue de l'IRES, n 66, p. 5-34, http://bit.ly/2J8zan1.

Gassier Y. (2020), « Si loin, si près des entreprises. Du projet syndical aux pratiques de représentation des salariés et chefs de très petites entreprises ", in Gassier Y., Giraud B. (dir.), Le travail syndical en actes. Faire adhérer, mobiliser, représenter, Villeneuve-d'Ascq, Presses universitaires du Septentrion, p. 188-205, https://hal.archives-ouvertes.fr/hal-02491247.

Gaxie D. (1978), Le cens caché. Inégalités culturelles et ségrégation politique, Paris, Éditions du Seuil.

Giraud B., Yon K., Béroud S. (2018), Sociologie politique du syndicalisme, Paris, Armand Colin.

Haute T. (2019a), Les salarié·e·s aux urnes : contribution à l'étude des ressorts collectifs et individuels des votes des salariés aux scrutins professionnels dans le secteur privé en France, Thèse de doctorat en science politique, Université Lille 2, http://www.theses.fr/2019LIL2D005.

Haute T. (2019b), « Le vote professionnel, entre rapport au syndicalisme et rapport au métier et à l'institution : le cas des enseignant·e.s du secteur public en France », Participations, n 25, p. 139-164, https://doi.org/10.3917/ parti.025.0139.

Haute T., Yon K. (2018), « Comprendre la construction sociale de la représentativité syndicale : un éclairage à partir des branches professionnelles », Travail et Emploi, n 154, p. 101-124, https://doi.org/10.4000/travailemploi.8282.

Lehingue P. (2011), Le vote : approches sociologiques de l'institution et des comportements électoraux, Paris, La Découverte.

Le-Ninivin D. (2018), " L'emploi dans les très petites entreprises fin 2016 », Dares Résultats, $\mathrm{n}^{\circ} 001$, janvier, https://dares.travail-emploi.gouv.fr/IMG/pdf/2018001.pdf.

Lescurieux M. (2019), « La représentation syndicale des femmes, de l'adhésion à la prise de responsabilités : une inclusion socialement sélective ", La Revue de l'IRES, n 98, p. 89-112, https://bit.ly/3bZelGg.

Malo M.A. (2006), « Temporary Workers and Direct Voting Systems for Workers' Representation », Economic and Industrial Democracy, n² 27, p. 505-535, https://doi.org/10.1177/0143831X06065966.

Mazaud C. (2012), "Artisan, de l'homme de métier au gestionnaire ? ", Travail et Emploi, n¹30, p. 9-20, https://doi.org/10.4000/travailemploi.5652.

Peugny C. (2015), " Pour une prise en compte des clivages au sein des classes populaires : la participation politique des ouvriers et des employés », Revue française de science politique, $\mathrm{n}^{\circ} 65, \mathrm{p} .735-759$, https://doi.org/10.3917/ rfsp.655.0735. 
Rey F. (2014), " La démocratie politique contre la démocratie sociale ? Analyse de la négociation sur la représentation des salariés de TPE ", Négociations, $\mathrm{n}^{\circ} 21$, p. 35-49, https://doi.org/10.3917/neg.021.0035.

Rey F. (2016), « Un droit universel à la représentation ? Un demi-siècle d'institutionnalisation du dialogue social territorial à destination des TPE », La Nouvelle Revue du Travail, $\mathrm{n}^{\circ}$ 8, https://doi.org/10.4000/nrt.2562.

Rey F., Borisova K., Thobois P. (2014), Construire un syndicalisme de proximité dans les petites entreprises. Stratégies, pratiques et actions syndicales en direction des salariés des petites entreprises : le cas de la CFDT, Rapport réalisé pour la CFDT dans le cadre de l'agence d'objectifs de l'Ires, mai, https://bit.ly/2UQ22GE.

Sin P., Grima F. (2019), « La militance syndicale dans la très petite entreprise : le cas du conseiller du salarié ", Relations Industrielles/Industrial Relations, $\mathrm{n}^{\circ} 74$, p. 66-88, https://doi.org/10.7202/1059465ar.

Subileau F. (1999), « Les élections prud'homales. Participation, représentativité, légitimité », Politix, n²47, p. 151-166, https://doi.org/10.3406/polix.1999.1797.

Tall A. (2018), « Le dialogue social dans les TPE fin 2016 », Dares Résultats, n 020, mai, https://dares.travail-emploi.gouv.fr/IMG/pdf/2018-020.pdf.

Tiberj V. (2018), « Le vote décentré ? Renouvellement générationnel et rapport à la participation électorale en France ", Revue française de science politique, $n^{\circ} 68$, p. 821-845, https://doi.org/10.3917/rfsp.685.0821.

Trémeau C. (2017), « De jeunes salariés confrontés à l'(in)justice du travail : recours aux prud'hommes et effets socialisateurs de l'épreuve judiciaire ", Politix, n 118, p. 157-181, https://doi.org/10.3917/pox.118.0157.

Trémeau C. (2019a), " Des "arrangements" à la confrontation. Les jeunes coiffeuses et ouvriers du bâtiment face à leur.s employeur's ", La Nouvelle Revue du Travail, n 15, https://doi.org/10.4000/nrt.5723.

Trémeau C. (2019b), « La distance de jeunes salariés vis-à-vis des "voies du collectif" », La Revue de I'IRES, n 99, p. 63-89, https://bit.ly/2JOgZ5U. 